Bull. Austral. Math. Soc.

$65 \mathrm{~F} 10,15 \mathrm{~A} 06$

VOL. 75 (2007) [289-298]

\title{
A CLASS OF ITERATION METHODS FOR THE MATRIX EQUATION
}

$$
A X B=C
$$

\section{Konghua Guo, XiYan Hu and Lei Zhang}

\begin{abstract}
An iteration method for the matrix equation $A X B=C$ is constructed. By this iteration method, the least-norm solution for the matrix equation can be obtained when the matrix equation is consistent and the least-norm least-squares solutions can be obtained when the matrix equation is not consistent. The related optimal approximation solution is obtained by this iteration method. A preconditioned method for improving the iteration rate is put forward. Finally, some numerical examples are given.
\end{abstract}

\section{INTRODUTION}

The matrix equation problem is an active research topic in computational mathematics, and has been widely applied in various areas, such as structural design, system identification, principal component analysis, exploration and remote sensing, biology, electricity, solid mechanics, molecular spectroscopy, structural dynamics, automatics control theory, vibration theory, and so on.

We use $R^{n}$ to denote the set of all real vectors of $n$ dimensions, $I_{n}$ the identity matrix of order $n$, and $R^{n \times m}$ all $n \times m$ real matrices. Let $\|A\|_{F}, A^{+}, A^{T}$ denote especially the Frobenius norm, the Moore-Penrose generalised inverse, and the transpose of a matrix $A$. $(\operatorname{tr}(A)$ means the trace of matrix $A, R(A)$ the column space of matrix $A), R^{\perp}(A)$ the orthogonal complement space of $R(A)$, and for any $A \in R^{m \times n}, B \in R^{n \times p}, A \otimes B$ means the Kronecker product of the matrices $A$ and $B$.

The following problems are considered in this paper.

Problem 1.1. Given $A \in R^{m \times n}, B \in R^{n \times p}, C \in R^{m \times p}$, find $X \in R^{n \times n}$, such that

$$
A X B=C
$$

Problem 1.2. Suppose Problem 1.1 is consistent, and its solution set is $S_{E}$, for $X_{0}$ $\in R^{n \times n}$. Find $\widehat{X} \in S_{E}$, such that

$$
\left\|\widehat{X}-X_{0}\right\|_{F}=\min _{X \in S_{E}}\left\|X-X_{0}\right\|_{F}
$$

Received 18th October, 2006

Research supported by National Natural Science Foundation of China(10571047) and Scientific Research Fund of Hunan Provincial Education Department(06C095)

Copyright Clearance Centre, Inc. Serial-fee code: 0004-9727/07 \$A2.00+0.00. 
In fact, Problem 1.2 is to find the optimal approximation solution to the given matrix $X_{0}$. In 1955 , Penrose obtained necessary and sufficient conditions for solving Problem 1.1 and the general expressions of the solution [10]. Since then Problem 1.1 has been considered in the case of some special solution structures, for example, symmetric, triangular or diagonal solution $X$. We can refer to Hua [3], Chu [2], Don [4], Magnus [6], Morris and Odell [8], Bjerhammer [1] for more details. Mitra [7] considered common solutions to a pair of linear matrix equations $A_{1} X B_{1}=C_{1}, A_{2} X B_{2}=C_{2}$. In these papers, the problem was discussed by using matrix decompositions such as the singular value decomposition, the generalised single valued decomposition, the quotient single valued decomposition and the canonical correlation decomposition. However, it is difficult to apply these methods to solving problems such as finding symmetric solutions of the matrix equation $A X B=C$. In 2005, Y.X. Peng put forward an iteration method for finding symmetric solutions of the matrix equation $A X B=C$ ([9]). The advantage of this iteration method is that when the problem is consistent, its solution can be obtained theoretically within a finite number of steps, and the disadvantage of the method is that the convergence rate can not be analysed.

In this paper, we construct a new iterative method for the matrix equation $A X B$ $=C$, by which we can obtain the least-norm solution of Problem1.1 when the problem is consistent and obtain the least-norm least-squares solution of Problem1.1 when the problem is not consistent. Furthermore, we show that the convergence rate of the method is related to the singular value of the matrix $A$, and so the iteration method can be improved by some preconditioned methods. When the solution set of Problem 1.1 is not empty, Problem 1.2 has a unique solution and we can obtain it by the iteration method.

The paper is organised as follows: In Section 2 we first introduce a new iterative method for finding the matrix equation $A X B=C$ and prove the convergence of the method. In Section 3 we solve Problem 1.2 by using this iteration method. In Section 4 we propose an improvement of the iteration method in order to increase the convergence rate. In the last section, we shall give some numerical examples to verify the method and compare the convergence rate between the original method and the improved method.

\section{The SOlution of Problem 1.1}

In this section, we shall introduce a new iteration method for solving Problem 1.1, and then we shall prove the convergence of the iteration method.

ITERATION METHOD 2.1.

stepl: Select $C_{0}=C, X_{0}=O$;

step2: Let $\alpha_{k}=\frac{\left\|A^{T} C_{k} B^{T}\right\|_{F}^{2}}{\left\|A A^{T} C_{k} B^{T} B\right\|_{F}^{2}}(k=0,1 ; 2, \ldots)$;

step3: Let $\Delta X_{k}=\alpha_{k} A^{T} C_{k} B^{T},(k=0,1,2, \ldots)$;

step4: If $\Delta X_{k}=0$, stop, otherwise, let $X_{k+1}=X_{k}+\Delta X_{k},(k=0,1,2, \ldots)$; 
step5: Let $C_{k+1}=C_{k}-A \Delta X_{k} B,(k=0,1,2, \ldots)$, goto step2.

Definition 2.1: Suppose $A, B \in R^{m \times n}$, then $\operatorname{tr}\left(A^{T} B\right)$ is called the inner product of the matrices $\mathrm{A}, \mathrm{B}$, denoted by $\langle A, B\rangle$.

Definition 2.2: Assume $A, B \in R^{m \times n}$. If $\langle A, B\rangle=0$, that is, $\operatorname{tr}\left(A^{T} B\right)=0$, then the matrices $A, B$ are called orthogonal each other.

Lemma 2.1. In the iteration method 2.1, the selection of $\alpha_{k}$ makes $\left\|C_{k+1}\right\|_{F}$ minimal, and make $C_{k+1}$ and $A \Delta X_{k} B$ orthogonal.

PROOF: For the iteration method 2.1, we have

$$
\begin{aligned}
\left\|C_{k+1}\right\|_{F}^{2} & =\left\langle C_{k}-\alpha_{k} A^{T} C_{k} B^{T}, C_{k}-\alpha_{k} A^{T} C_{k} B^{T}\right\rangle \\
& =\left\|C_{k}\right\|_{F}^{2}-2 \alpha_{k}\left\langle C_{k}, A A^{T} C_{k} B^{T} B\right\rangle+\alpha_{k}^{2}\left\|A A^{T} C_{k} B^{T} B\right\|_{F}^{2}
\end{aligned}
$$

From the above expression, we know that the necessary and sufficient conditions of making $\left\|C_{k+1}\right\|_{F}$ the minimal is that

$$
\alpha_{k}=\frac{\left\|A^{T} C_{k} B^{T}\right\|_{F}^{2}}{\left\|A A^{T} C_{k} B^{T} B\right\|_{F}^{2}}
$$

On the other hand, Let $\left\langle C_{k+1}, A \Delta X_{k} B\right\rangle=0$, we also have that

$$
\alpha_{k}=\frac{\left\|A^{T} C_{k} B^{T}\right\|_{F}^{2}}{\left\|A A^{T} C_{k} B^{T} B\right\|_{F}^{2}} .
$$

Hence, in Iteration method 2.1, selecting

$$
\alpha_{k}=\left\|A^{T} C_{k} B^{T}\right\|_{F}^{2} /\left\|A A^{T} C_{k} B^{T} B\right\|_{F}^{2}
$$

will make $C_{k+1}$ and $A \Delta X_{k} B$ orthogonal.

LEMMA 2.2. In the iteration method 2.1, we have $\left\|C_{k+1}\right\|_{F}^{2}=\left\|C_{k}\right\|_{F}^{2}-\left\|A \Delta X_{k} B\right\|_{F}^{2}$

Proof: From the step5 of Iteration method 2.1, we have $C_{k}=C_{k+1}+A \Delta X_{k} B$, and so, $\left\|C_{k}\right\|_{F}^{2}=\left\|C_{k+1}+A \Delta X_{k} B\right\|_{F}^{2}$, according to Lemma 2.1, then we have

$$
\left\|C_{k}\right\|_{F}^{2}=\left\|C_{k+1}\right\|_{F}^{2}+\left\|A \Delta X_{k} B\right\|_{F}^{2} .
$$

Hence,

$$
\left\|C_{k+1}\right\|_{F}^{2}=\left\|C_{k}\right\|_{F}^{2}-\left\|A \Delta X_{k} B\right\|_{F}^{2} .
$$

Definition 2.3: For $A=\left(a_{i j}\right)_{m \times n} \in R^{m \times n}$, denote by $\operatorname{vec}(A)$ the following vector containing all the entries of matrix $A$ :

$$
\operatorname{vec}(A)=\left[a_{11}, \ldots, a_{1 n}, a_{21}, \ldots, a_{2 n}, \cdots, a_{m a}, \ldots, a_{m n}\right]^{T},
$$

then $\operatorname{vec}(A)$ is called straightening of the matrix $A$. 
It is evident that the transform $A \longrightarrow \operatorname{vec}(A)$ gives a linear isomorph of $R^{m \times n}$ $\longrightarrow R^{m n}$.

Lemma 2.3. ([5]) For any matrices $A, B$ and $C$ in suitable size, we have

$$
\operatorname{vec}(A+B)=\operatorname{vec}(A)+\operatorname{vec}(B), \operatorname{vec}(A B C)=\left(A \otimes C^{T}\right) \operatorname{vec}(B)
$$

LEMma 2.4. Suppose that the consistent system of linear equations $M y=b$ has a solution $y_{0} \in R\left(M^{T}\right)$, then $y_{0}$ is the least-norm solution of the system of linear equations.

Proof: We decompose the matrix $M$ by single valued decomposition:

$$
M=U\left(\begin{array}{cc}
\Sigma & 0 \\
0 & 0
\end{array}\right) V^{T}=U_{1} \Sigma V_{1}^{T}
$$

where $U=\left(U_{1}, U_{2}\right)$ and $V=\left(V_{1}, V_{2}\right)$ are orthogonal matrices. Then the Moore-Penrose generalised inverse of matrix $M$ is

$$
M^{+}=V_{1} \Sigma^{+} U_{1}^{T}
$$

and the general solution of the system of linear equations $M y=b$ is

$$
y=M^{+} b+\left(I-M^{+} M\right) z
$$

where $z$ is an arbitrary vector with suitable size.

Since $M^{+}=V_{1} \Sigma^{+} U_{1}^{T} \in R\left(V_{1}\right),\left(I-M^{+} M\right) z=\left(I-V_{1} V_{1}^{T}\right) z=V_{2} V_{2}^{T} z \in R\left(V_{2}\right)$, and $V_{2}$ and $V_{1}$ are orthogonal each other; that is, $\operatorname{tr}\left(V_{2}^{T} V_{1}\right)=0$, then $M^{+} b$ is the least-norm solution of the system of linear equations $M y=b$.

On the other hand, $M^{T}=V_{1} \Sigma U_{1}^{T}$, the solution $y_{0} \in R\left(M^{T}\right)$, therefore $y_{0}$ is the least-norm solution of the system of linear equations $M y=b$.

Obviously, the set of solutions of the system of linear equations $M y=b$ is closed convex, and so the least-norm solution of the system is unique.

Similarly, we have the following lemma.

LEMMA 2.5. Suppose that the inconsistent system of linear equations $M y=b$ has a solution $y_{0} \in R\left(M^{T}\right)$, then $y_{0}$ is the least-norm least-squares solution of the system of linear equations, and the solution is unique.

LEMMA 2.6. ([11]) The matrix equation $A X B=C$ has a unique least-norm solution $X=A^{+} C B^{+}$when the equation is consistent, and has a unique least-norm least-squares solution $X=A^{+} C B^{+}$when the equation is not consistent.

Definition 2.4: Let $A, B \in R^{m \times n}$. If $\cos \theta=\langle A, B\rangle /\left(\|A\|_{F} \cdot\|B\|_{F}\right)(0 \leqslant \theta$ $\leqslant \pi$ ), then $\theta$ is called the included angle of the matrices $A, B$. 
THEOREM 2.1. The iteration method 2.1 is convergent. Let the maximum singular value and the minimum singular value of the matrix $A$ be $\sigma_{1}, \sigma_{r}$, the maximum singular value and the minimum singular value of the matrix $B$ be $\lambda_{1}, \lambda_{s}$. Then the convergence rate of the iteration method 2.1 is no less than $-0.5 \ln \left(1-\left(\sigma_{r}^{2} \lambda_{s}^{2}\right) /\left(\sigma_{1}^{2} \lambda_{1}^{2}\right)\right)$.

Proof: . Suppose $\operatorname{rank}(A)=r, \operatorname{rank}(B)=s$, and the singular value decompositions of matrix $A$ and matrix $B$ are

$$
A=U D V^{T}=U_{1} \Sigma V_{1}^{T}, B=P E Q^{T}=P_{1} \Lambda Q_{1}^{T}
$$

where $U=\left(U_{1}, U_{2}\right), V=\left(V_{1}, V_{2}\right), P=\left(P_{1}, P_{2}\right)$, and $Q=\left(Q_{1}, Q_{2}\right)$ are orthogonal matrices, $D=\left(\begin{array}{ll}\Sigma & O \\ O & O\end{array}\right), E=\left(\begin{array}{cc}\Lambda & O \\ O & O\end{array}\right), \Sigma=\operatorname{diag}\left(\sigma_{1}, \sigma_{2}, \ldots, \sigma_{r}\right), \sigma_{i}(i=1,2, \ldots, r)$ are the singular values of matrix $A ; \Lambda=\operatorname{diag}\left(\lambda_{1}, \lambda_{2}, \ldots, \lambda_{s}\right), \lambda_{i}(i=1,2, \ldots, s)$ are the singular values of matrix $B$.

If Problem 1.1 is consistent, then for $C_{k}$ there exits a matrix $G$ which makes $C_{k}$ $=U G Q^{T}$, where $G=\left(g_{i j}=\left(g_{1}, \ldots, g_{n}\right), g_{i} \in R^{n},(i=1,2, \ldots, n), g_{i}=0, i>s . g_{i j}\right.$ $\in R,(i, j=1,2, \ldots, n), g_{i j}=0, i>r, j>s$

Let $\theta$ be the included angle of $C_{k}$ and $A \Delta X_{k} B$, then we have that

$$
\begin{aligned}
\cos (\theta) & =\frac{\left(C_{k}, A \Delta X_{k} B\right)}{\left\|C_{k}\right\|_{F} \cdot\left\|A \Delta X_{k} B\right\|_{F}}=\frac{\left\|A^{T} C_{k} B^{T}\right\|_{F}^{2}}{\left\|C_{k}\right\|_{F} \cdot\left\|A A^{T} C_{k} B^{T} B\right\|_{F}} \\
& =\frac{\left\|V D^{T} U^{T} U G Q^{T} Q E^{T} P\right\|_{F}^{2}}{\left\|U G Q^{T}\right\|_{F} \cdot\left\|U D V^{T} V D^{T} U^{T} U G Q^{T} Q E^{T} P^{T} P E Q^{T}\right\|_{F}} \\
& =\frac{\|\left. D^{T} G E\right|_{F} ^{2}}{\|G\|_{F} \cdot\left\|D D^{T} G E^{T} E\right\|_{F}}=\frac{\operatorname{tr}\left(E G^{T} D D^{T} G E^{T}\right)}{\left(\operatorname{tr}\left(G^{T} G\right)\right)^{1 / 2} \cdot\left(\operatorname{tr}\left(E^{T} E G^{T} D D^{T} D D^{T} G E^{T} E\right)\right)^{1 / 2}} \\
& =\frac{\sum_{j=1}^{s} \sum_{i=1}^{r} \sigma_{i}^{2} \lambda_{j}^{2} g_{i j}^{2}}{\left(\sum_{i=1}^{s} g_{i}^{T} g_{i}\right)^{1 / 2} \cdot\left(\sum_{j=1}^{s} \sum_{i=1}^{r} \sigma_{i}^{4} \lambda_{j}^{4} g_{i j}^{2}\right)^{\frac{1}{2}}} \\
& \geqslant \frac{\sum_{j=1}^{s} \sum_{i=1}^{r} \sigma_{i}^{2} \lambda_{j}^{2} g_{i j}^{2}}{\left.\left(\sum_{i=1}^{s} g_{i}^{T} g_{i}\right)^{1 / 2} \cdot\left(\sum_{j=1}^{s} \sum_{i=1}^{r} \sigma_{i}^{2} \lambda_{j}^{2} \lambda_{i j}^{2}\right)^{1 / 2} g_{i j}^{2}\right)^{1 / 2}}
\end{aligned}
$$

Notice that $\left\|C_{k}\right\|_{F}=\left\|C_{k+1}\right\|_{F}+\left\|A \Delta X_{k} B\right\|_{F}$, then we have

$$
\left\|C_{k+1}\right\|_{F}=\left\|C_{k}\right\|_{F} \sin (\theta) \leqslant \sqrt{1-\frac{\sigma_{\tau}^{2} \lambda_{s}^{2}}{\sigma_{1}^{2} \lambda_{1}^{2}}} .
$$

Therefore, Iteration method 2.1 is convergent, and the convergence rate of the iteration $\operatorname{method}(2.1)$ is no less than $-0.5 \ln \left(1-\left(\sigma_{r}^{2} \lambda_{s}^{2}\right) /\left(\sigma_{1}^{2} \lambda_{1}^{2}\right)\right)$. 
THEOREM 2.2. The iteration method 2.1 will converge to the least-norm solution of Problem 1.1 when the problem is consistent and will converge to the least-norm leastsquare solution of Problem 1.1 when the problem is not consistent.

Proof: According to Theorem 2.1, if Problem 1.1 is consistent, we can obtain a solution $X^{*}$ by Iteration method 2.1 , and the solution $X^{*}$ can be represented as that

$$
X^{*}=A^{T} Y B^{T}
$$

In the sequel, we shall prove that the $X^{*}$ is just the least-norm solution of Problem 1.1 .

Denote $\operatorname{vec}(X)=x, \operatorname{vec}\left(X^{*}\right)=x^{*}, \operatorname{vec}(Y)=y, \operatorname{vec}(C)=b$, then the matrix equations $A X B=C$ is equivalent to the system of linear equations

$$
\left(A \otimes B^{T}\right) x=b
$$

Notice that

$$
\begin{aligned}
x^{*} & =\operatorname{vec}\left(X^{*}\right)=\operatorname{vec}\left(A^{T} Y B^{T}\right)=\left(A^{T} \otimes B\right) y \\
& =\left(A \otimes B^{T}\right)^{T} y \in R\left(\left(A \otimes B^{T}\right)^{T}\right)
\end{aligned}
$$

So $x^{*}$ is the least-norm solution of the system of linear equation 2.1 by Lemma 2.4 , Since the vector operator is isomorphic, $X^{*}$ is the unique least-norm solution of Problem 1.1 .

If Problem 1.1 is not consistent, let $C=C^{(1)}+C^{(2)}$, where $C^{(1)} \in R(A)$ and $C^{(2)}$ $\in R^{\perp}(A)$. For any $X \in R^{n \times n}, C^{(1)}-A X B \in R(A)$, and $C^{(1)}-A X B$ is orthogonal with $C^{(2)}$, so we have that

$$
\|C-A X B\|_{F}^{2}=\left\|C^{(1)}-A X B+C^{(2)}\right\|_{F}^{2}=\left\|C^{(1)}-A X B\right\|_{F}^{2}+\left\|C^{(2)}\right\|_{F}^{2}
$$

which means that the sufficient and necessary condition of $X$ being the least-squares solution of $A X B=C$ is that $X$ is the solution of consistent equation $A X B=C^{(1)}$.

From the step5 of Iteration method 2.1, we have

$$
C_{k+1}^{(1)}+C_{k+1}^{(2)}=C_{0}^{(1)}+C_{0}^{(2)}-A X_{k+1} B
$$

Noticing that $A X_{k+1} B \in R(A)$, we know $C_{k+1}^{(2)}=C_{0}^{(2)}$, and (2.3) is equivalent to

$$
C_{k+1}^{(1)}=C_{0}^{(1)}-A X_{k+1} B
$$

Thus the iteration process is conducted in $R(A)$. Then from the iteration method 2.1 , we can obtain the least-norm solution of the consistent equation $A X B=C^{(1)}$. It means that the iteration method will converge to the unique least-norm least-squares solution of Problem 1.1 when the problem is not consistent. 


\section{The Solution of Problem 1.2}

When Problem 1.1 is solvable, it is easy to test that $S_{E}$ is a closed convex set. Hence we know that for the given $X_{0} \in R^{n \times n}$, we can find a unique $\widehat{X} \in S_{E}$ which will make $\left\|\widehat{X}-X_{0}\right\|_{F}=\min _{X \in S_{E}}\left\|X-X_{0}\right\|_{F}$. Next we give the iteration method which find the $\widehat{X} \in S_{E}$

If $S_{E}$ is not empty, for any $X \in S_{E}$,

$$
A X B=C \Leftrightarrow A\left(X-X_{0}\right) B=C-A X_{0} B
$$

Let $X^{*}=X-X_{0}, C^{*}=C-A X_{0} B$, then solving the problem 1.2 is equivalent to finding the least-norm solution $\widetilde{X}^{*}$ of the consistent matrix equation $A X^{*} B=C^{*}$, which can be obtained by using Iteration method 2.1, and the solution of the problem 1.2 can be represented as $\widehat{X}=\widetilde{X}^{*}+X_{0}$.

\section{THE IMPROVEMENTS OF THE ITERATION METHOD}

From Theorem 2.1, if the ratio $\left(\sigma_{r} \lambda_{s}\right) /\left(\sigma_{1} \lambda_{1}\right)$ is near to 1 , then the convergence of Iteration 2.1 will be fast, but if $\sigma_{1} \lambda_{1}>\sigma_{r} \lambda_{s}$, the convergence of Iteration Method 2.1 may be slow. To improve its convergence rate we may deal with the equation before solving it by using preconditioning methods.

In this paper, we adopt polynomial preconditioning methods to improve the convergence rate, that is, we transform the original equation $A X B=C$ to the equation $C(A) A X C(B)=C(A) C C(B)$, where $C(A), C(B)$ are polynomial on $A, B$ with low order.

In next section, we shall give some example to verify Iteration method 2.1 and compare the convergence rate between original iteration method and preconditioned method.

\section{EXAMPLE}

In this section, we denote $t$ as the computing time (unit:second), and $k$ as the number of iterations. The computations were performed using MATLAB, version 6.5.1, under the operation system of Windows $\mathrm{Me}$, and the CPU rate of the machine is $2.40 \mathrm{GHz}$.

EXAMPLE 1. Let




$B=A$, and let $C=$

$\left.\begin{array}{|lllllllllll}\mathbf{9 0 4 . 6 9 3 6} & \mathbf{9 1 3 . 2 0 0 6} & \mathbf{9 0 7 . 3 6 1 7} & \mathbf{8 8 1 . 3 1 8 7} & \mathbf{8 2 0 . 4 2 5 6} & \mathbf{6 5 5 . 7 4 0 0} & \mathbf{7 5 1 . 2 4 1 3} & \mathbf{8 1 0 . 0 6 7 2} & \mathbf{8 5 3 . 1 0 0 9} & \mathbf{8 8 3 . 1 0 3 9} & \mathbf{8 9 8 . 1 3 8 1} \\ \mathbf{9 2 3 . 8 0 0 8} & \mathbf{9 3 2 . 8 1 3 3} & \mathbf{9 2 7 . 1 3 9 9} & \mathbf{9 0 0 . 7 0 9 4} & \mathbf{8 3 8 . 2 3 8 7} & \mathbf{6 6 7 . 1 6 7 5} & \mathbf{7 6 3 . 4 9 3 7} & \mathbf{8 2 6 . 0 1 5 8} & \mathbf{8 7 0 . 3 4 2 7} & \mathbf{9 0 1 . 3 3 5 1} & \mathbf{9 1 6 . 9 7 4 3} \\ \mathbf{9 1 1 . 8 7 5 8} & \mathbf{9 2 1 . 1 3 3 3} & \mathbf{9 1 5 . 9 3 1 3} & \mathbf{8 9 0 . 2 0 9 4} & \mathbf{8 2 8 . 5 4 7 9} & \mathbf{6 5 6 . 6 6 4 7} & \mathbf{7 5 4 . 3 2 5 5} & \mathbf{8 1 4 . 3 7 1 5} & \mathbf{8 5 8 . 4 0 3 8} & \mathbf{8 8 9 . 2 9 3 1} & \mathbf{9 0 5 . 0 1 9 2} \\ \mathbf{8 9 2 . 7 8 5 2} & \mathbf{9 0 1 . 9 1 0 9} & \mathbf{8 9 7 . 0 8 1 4} & \mathbf{8 7 2 . 4 3 8 8} & \mathbf{8 1 2 . 9 1 4 9} & \mathbf{6 4 4 . 4 8 4 5} & \mathbf{7 3 9 . 5 2 2 7} & \mathbf{7 9 7 . 8 9 3 4} & \mathbf{8 4 0 . 6 9 5 3} & \mathbf{8 7 0 . 7 5 3 4} & \mathbf{8 8 6 . 1 3 1 3} \\ \mathbf{8 3 5 . 6 3 3 3} & \mathbf{8 4 3 . 6 0 1 8} & \mathbf{8 3 8 . 9 9 8 9} & \mathbf{8 1 6 . 6 9 3 0} & \mathbf{7 6 3 . 3 7 2 9} & \mathbf{6 1 1 . 6 5 0 9} & \mathbf{6 9 7 . 6 7 3 0} & \mathbf{7 5 0 . 4 7 2 5} & \mathbf{7 8 9 . 0 7 5 7} & \mathbf{8 1 6 . 0 6 8 6} & \mathbf{8 2 9 . 8 1 0 6} \\ \mathbf{7 2 5 . 1 2 0 6} & \mathbf{7 2 8 . 6 1 1 7} & \mathbf{7 2 2 . 1 1 7 6} & \mathbf{7 0 2 . 2 3 6 5} & \mathbf{6 6 0 . 9 5 9 9} & \mathbf{5 6 3 . 0 6 9 1} & \mathbf{6 2 6 . 5 1 0 0} & \mathbf{6 6 5 . 8 3 2 0} & \mathbf{6 9 4 . 0 4 8 9} & \mathbf{7 1 2 . 9 4 2 1} & \mathbf{7 2 1 . 5 5 7 3} \\ \mathbf{7 8 4 . 2 7 5 8} & \mathbf{7 8 9 . 1 8 1 6} & \mathbf{7 8 2 . 7 5 5 0} & \mathbf{7 6 0 . 8 8 0 6} & \mathbf{7 1 3 . 6 2 7 5} & \mathbf{5 9 5 . 9 0 1 1} & \mathbf{6 6 9 . 1 3 2 2} & \mathbf{7 1 4 . 4 1 6 1} & \mathbf{7 4 7 . 1 3 0 2} & \mathbf{7 6 9 . 3 5 3 5} & \mathbf{7 7 9 . 8 5 3 2} \\ \mathbf{8 3 4 . 0 0 5 5} & \mathbf{8 4 0 . 0 3 5 1} & \mathbf{8 3 3 . 5 9 7 8} & \mathbf{8 0 9 . 9 8 7 2} & \mathbf{7 5 7 . 7 1 5 7} & \mathbf{6 2 3 . 9 7 1 9} & \mathbf{7 0 5 . 2 6 4 7} & \mathbf{7 5 5 . 4 7 2 4} & \mathbf{7 9 1 . 8 9 5 3} & \mathbf{8 1 6 . 8 5 1 8} & \mathbf{8 2 8 . 8 7 7 9} \\ \mathbf{8 6 2 . 2 4 1 8} & \mathbf{8 6 9 . 0 4 0 9} & \mathbf{8 6 2 . 6 8 9 7} & \mathbf{8 3 8 . 1 0 5 4} & \mathbf{7 8 2 . 7 8 2 5} & \mathbf{6 3 8 . 6 3 4 1} & \mathbf{7 2 4 . 9 4 6 9} & \mathbf{7 7 8 . 2 0 8 0} & \mathbf{8 1 6 . 9 4 6 4} & \mathbf{8 4 3 . 6 3 3 6} & \mathbf{8 5 6 . 6 5 5 6} \\ \mathbf{8 9 0 . 7 2 3 2} & \mathbf{8 9 8 . 3 4 0 8} & \mathbf{8 9 2 . 1 0 5 8} & \mathbf{8 6 6 . 5 3 4 3} & \mathbf{8 0 8 . 0 4 0 9} & \mathbf{6 5 3 . 1 2 1 6} & \mathbf{7 4 4 . 5 8 4 5} & \mathbf{8 0 0 . 9 8 0 2} & \mathbf{8 4 2 . 1 0 4 3} & \mathbf{8 7 0 . 5 8 5 4} & \mathbf{8 8 4 . 6 4 7 2} \\ \mathbf{8 9 5 . 9 2 6 3} & \mathbf{9 0 3 . 8 1 6 5} & \mathbf{8 9 7 . 6 5 1 6} & \mathbf{8 7 1 . 8 1 1 4} & \mathbf{8 1 2 . 3 6 1 3} & \mathbf{6 5 4 . 3 1 1 3} & \mathbf{7 4 7 . 2 0 8 9} & \mathbf{8 0 4 . 4 8 2 9} & \mathbf{8 4 6 . 2 9 1 6} & \mathbf{8 7 5 . 3 0 3 9} & \mathbf{8 8 9 . 6 8 3 6}\end{array}\right)$

(1) Find the least-norm solution of Problem 1.1.

(2) Let $S_{E}$ denote the set of all solutions of the matrix equation $A X B=C$, suppose $X_{0}=$.

\begin{tabular}{|c|c|c|c|c|c|c|c|c|c|c|}
\hline 1.3701 & -4.8758 & 2.1230 & 0.7781 & 3.6417 & 2.3740 & 2.4194 & 4.3440 & 0.3185 & 3.9318 & -3.0110 \\
\hline-0.6231 & -1.6490 & 2.3485 & 1.0349 & -5.6921 & -4.2331 & 0.0966 & -1.6502 & 3.3918 & 3.8883 & -3.0388 \\
\hline-2.7381 & 2.0104 & -1.1974 & -0.5989 & 1.7949 & 5.4612 & -2.5518 & -4.5650 & 2.7999 & 3.0393 & -3.0322 \\
\hline-3.9526 & 3.6712 & -2.2277 & -1.0113 & 4.2979 & -5.3661 & 1.5700 & 2.0865 & 0.2286 & 1.2513 & -3.0349 \\
\hline-4.1968 & 2.2302 & 0.6011 & 0.5023 & -4.9471 & 3.5160 & 1.1593 & 4.0253 & -3.4762 & -1.1058 & -3.0181 \\
\hline-2.8254 & -3.3452 & 4.0723 & 1.6831 & 2.3066 & -1.7267 & -3.1806 & -2.9813 & -3.6434 & -3.4390 & -2.9897 \\
\hline-0.5413 & -3.8440 & -3.5421 & -3.5699 & -0.8585 & 0.4972 & 4.6667 & -2.1376 & 1.8821 & -4.4475 & -2.9977 \\
\hline $2.045 \theta$ & 0.3452 & -2.6328 & 5.0286 & 0.0968 & 0.0902 & -3.3274 & 3.0677 & 4.1941 & -3.8691 & -3.0076 \\
\hline 3.8297 & 3.4886 & 5.0435 & -5.3804 & 0.3600 & 0.1758 & -1.0414 & 1.1182 & 1.6169 & -2.1467 & -3.0099 \\
\hline 4.2991 & 3.2883 & 0.0882 & 4.3508 & 0.3813 & 0.1274 & 4.5694 & -3.5804 & -2.9543 & 0.3093 & -3.0140 \\
\hline 3.3747 & -1.3949 & -4.6599 & -2.8194 & -1.3521 & -1.0905 & -4.1776 & 0.2709 & -4.6121 & 2.4728 & -3.0121 \\
\hline
\end{tabular}

find the solution of Problem 1.2.

(1) At first, we find the least-norm solution of Problem 1.1 by iteration method 2.1. Let $\varepsilon=1.0 e-10$, and when $\left\|\Delta X_{k}\right\|<\varepsilon$, stop the iteration, then we have that

$$
X=\left(\begin{array}{llllllllllll}
1.0000 & 0.5000 & 0.3333 & 0.2600 & 0.2000 & 0.1667 & 0.1429 & 0.1250 & 0.1111 & 0.1000 & 0.0909 \\
0.5000 & 0.3333 & 0.2500 & 0.2000 & 0.1667 & 0.1429 & 0.1250 & 0.1111 & 0.1000 & 0.0909 & 0.0833 \\
0.3333 & 0.2500 & 0.2000 & 0.1667 & 0.1429 & 0.1250 & 0.1111 & 0.1000 & 0.0909 & 0.0833 & 0.0769 \\
0.2500 & 0.2000 & 0.1667 & 0.1429 & 0.1250 & 0.1111 & 0.1000 & 0.0909 & 0.0833 & 0.0769 & 0.0714 \\
0.2000 & 0.1667 & 0.1429 & 0.1250 & 0.1111 & 0.1000 & 0.0909 & 0.0833 & 0.0769 & 0.0714 & 0.0667 \\
0.1667 & 0.1429 & 0.1250 & 0.1111 & 0.1000 & 0.0909 & 0.0833 & 0.0769 & 0.0714 & 0.0687 & 0.0625 \\
0.1429 & 0.1250 & 0.1111 & 0.1000 & 0.0909 & 0.0833 & 0.0769 & 0.0714 & 0.0667 & 0.0625 & 0.0588 \\
0.1250 & 0.1111 & 0.1000 & 0.0909 & 0.0833 & 0.0769 & 0.0714 & 0.0667 & 0.0625 & 0.0588 & 0.0556 \\
0.1111 & 0.1000 & 0.0909 & 0.0833 & 0.0769 & 0.0714 & 0.0667 & 0.0625 & 0.0588 & 0.0556 & 0.0526 \\
0.1000 & 0.0909 & 0.0833 & 0.0769 & 0.0714 & 0.0667 & 0.0625 & 0.0588 & 0.0556 & 0.0526 & 0.0500 \\
0.0909 & 0.0833 & 0.0769 & 0.0714 & 0.0667 & 0.0625 & 0.0588 & 0.0556 & 0.0526 & 0.0500 & 0.0476
\end{array}\right)
$$

where, $\sigma_{r} / \sigma_{1}=\lambda_{s} / \lambda_{1}=4.4456 / 81.1530, t=7.2210, k=6756$. In comparison, by using the iteration method from the paper [9], we can get the same result with $t=28.6520$, $k=18317$.

Secondly, by using preconditioned iteration method, let $C(A) A=I_{11}-4 \times(0.001$ $\left.\times A-I_{11}\right)^{3}+3 \times(0.001 \times A)^{2}$, denote $C(A) A$ as $\widetilde{A}, C(A) C$ as $\widetilde{C}$, solving the equation $\widetilde{A} X \widetilde{A}=\widetilde{C}$, we can obtain the same $X$ with the original method, but where, $\sigma_{r} / \sigma_{1}$ $=\lambda_{s} / \lambda_{1}=4.1228 / 5.1832, t=0.1000, k=17$. And under the same situation, by using the iteration method from the paper [9], we find that the iteration is not convergent.

(2) Denotes the set of all solutions of the matrix equation $A X B=C$ in this example as $S_{E}$. In order to find the optimal approximate solution to a given matrix $X_{0}$, let $X^{*}=X-X_{0} C^{*}=C-A X_{0} B$, and $\varepsilon=1.0 e-10$, by iteration method 2.1 , when $\left\|\Delta X_{k}\right\|<\varepsilon$, stop the iteration, then we can obtain the least-norm solution $\tilde{X}^{*}$ of the 
consistent matrix equation $A X^{*} B=C^{*}$, and so the optimal approximation solution $\widehat{X}$ to the given matrix $X_{0}$ is that

$$
\widehat{X}=\widetilde{X}^{*}+X_{0}=\left(\begin{array}{lllllllllll}
1.0000 & 0.3000 & 0.3333 & 0.2500 & 0.2000 & 0.1667 & 0.1429 & 0.1250 & 0.1111 & 0.1000 & 0.0909 \\
0.5000 & 0.3333 & 0.2500 & 0.2000 & 0.1667 & 0.1429 & 0.1250 & 0.1111 & 0.1000 & 0.0909 & 0.0833 \\
0.3333 & 0.2500 & 0.2000 & 0.1667 & 0.1429 & 0.1250 & 0.1111 & 0.1000 & 0.0909 & 0.0833 & 0.0769 \\
0.2500 & 0.2000 & 0.1667 & 0.1429 & 0.1250 & 0.1111 & 0.1000 & 0.0909 & 0.0833 & 0.0769 & 0.0714 \\
0.2000 & 0.1667 & 0.1429 & 0.1250 & 0.1111 & 0.1000 & 0.0909 & 0.0833 & 0.0769 & 0.0714 & 0.0667 \\
0.1667 & 0.1429 & 0.1250 & 0.1111 & 0.1000 & 0.0909 & 0.0833 & 0.0769 & 0.0714 & 0.0667 & 0.0625 \\
0.1429 & 0.1250 & 0.1111 & 0.1000 & 0.0909 & 0.0833 & 0.0769 & 0.0714 & 0.0667 & 0.0625 & 0.0588 \\
0.1250 & 0.1111 & 0.1000 & 0.0909 & 0.0833 & 0.0769 & 0.0714 & 0.0667 & 0.0625 & 0.0588 & 0.0556 \\
0.1111 & 0.1000 & 0.0909 & 0.0833 & 0.0769 & 0.0714 & 0.0667 & 0.0625 & 0.0588 & 0.0556 & 0.0526 \\
0.1000 & 0.0909 & 0.0833 & 0.0769 & 0.0714 & 0.0667 & 0.0625 & 0.0588 & 0.0556 & 0.0526 & 0.0500 \\
0.0909 & 0.0833 & 0.0769 & 0.0714 & 0.0667 & 0.0625 & 0.0588 & 0.0556 & 0.0526 & 0.0500 & 0.0476
\end{array}\right)
$$

ExAMPLE 2. Let $A, B$ is as the same matrix as of the Example 1,

$$
\left.\begin{array}{cccccccccccc}
112.9234 & 101.6547 & \mathbf{9 7 . 0 8 5 0} & \mathbf{9 2 . 5 4 6 9} & \mathbf{8 5 . 4 0 8 7} & \mathbf{6 8 . 6 3 6 4} & \mathbf{7 7 . 4 2 0 7} & \mathbf{8 2 . 7 6 3 9} & \mathbf{8 6 . 6 5 3 1} & \mathbf{8 9 . 3 2 8 2} & \mathbf{9 0 . 5 8 4 5} \\
\mathbf{1 0 2 . 7 0 9 8} & \mathbf{9 9 . 8 7 4 3} & \mathbf{9 7 . 4 2 7 1} & \mathbf{9 3 . 6 8 3 8} & \mathbf{8 6 . 7 5 8 7} & \mathbf{6 9 . 3 0 9 1} & \mathbf{7 8 . 6 3 2 2} & \mathbf{8 4 . 3 0 7 1} & \mathbf{8 8 . 4 4 3 4} & \mathbf{9 1 . 3 0 6 6} & \mathbf{9 2 . 6 8 6 5} \\
\mathbf{9 7 . 5 4 2 0} & \mathbf{9 6 . 8 3 3 4} & \mathbf{9 5 . 2 6 9 0} & \mathbf{9 2 . 0 0 5 2} & \mathbf{8 5 . 3 7 2 8} & \mathbf{6 7 . 9 0 3 3} & \mathbf{7 7 . 2 9 8 2} & \mathbf{8 3 . 0 1 8 3} & \mathbf{8 7 . 1 9 3 4} & 90.0975 & \mathbf{9 1 . 5 2 2 7} \\
\mathbf{9 3 . 6 9 4 2} & \mathbf{9 3 . 8 0 5 4} & \mathbf{9 2 . 6 8 8 6} & \mathbf{8 9 . 7 6 1 0} & \mathbf{8 3 . 4 7 3 6} & \mathbf{6 6 . 4 0 6 3} & \mathbf{7 5 . 6 2 7 8} & \mathbf{8 1 . 2 4 2 6} & \mathbf{8 5 . 3 4 1 2} & \mathbf{8 8 . 1 9 7 7} & \mathbf{8 9 . 6 1 3 9} \\
\mathbf{8 6 . 9 2 6 8} & \mathbf{8 7 . 2 9 2 7} & \mathbf{8 6 . 4 1 1 7} & \mathbf{8 3 . 8 4 9 0} & \mathbf{7 8 . 2 6 3 9} & \mathbf{6 2 . 9 1 0 7} & \mathbf{7 1 . 2 8 9 2} & \mathbf{7 6 . 3 8 9 0} & \mathbf{8 0 . 1 0 0 0} & \mathbf{8 2 . 6 7 4 9} & \mathbf{8 3 . 9 4 6 3} \\
\mathbf{7 5 . 4 4 8 3} & \mathbf{7 5 . 3 9 1 8} & \mathbf{7 4 . 4 0 5 9} & \mathbf{7 2 . 1 5 3 7} & \mathbf{6 7 . 8 2 2 0} & \mathbf{5 7 . 8 8 6 0} & \mathbf{6 4 . 0 4 3 5} & \mathbf{6 7 . 8 2 4 2} & \mathbf{7 0 . 5 1 8 9} & \mathbf{7 2 . 3 0 1 4} & \mathbf{7 3 . 0 7 3 6} \\
\mathbf{8 0 . 6 7 8 2} & \mathbf{8 0 . 9 8 0 4} & \mathbf{8 0 . 1 2 5 5} & \mathbf{7 7 . 7 5 4 7} & \mathbf{7 2 . 8 7 9 1} & \mathbf{6 0 . 9 9 0 8} & \mathbf{6 8 . 1 6 8 6} & \mathbf{7 2 . 5 7 6 5} & \mathbf{7 5 . 7 4 5 3} & \mathbf{7 7 . 8 8 0 0} & \mathbf{7 8 . 8 3 5 6} \\
\mathbf{8 5 . 1 3 1 4} & \mathbf{8 5 . 6 9 8 5} & \mathbf{8 4 . 9 3 2 5} & \mathbf{8 2 . 4 4 8 1} & \mathbf{7 7 . 1 1 1 7} & \mathbf{6 3 . 6 4 6 5} & \mathbf{7 1 . 6 6 3 0} & \mathbf{7 6 . 5 8 8 0} & \mathbf{8 0 . 1 4 7 4} & \mathbf{8 2 . 5 7 1 1} & \mathbf{8 3 . 7 1 1 6} \\
\mathbf{8 7 . 5 6 8 8} & \mathbf{8 8 . 3 1 8 4} & \mathbf{8 7 . 6 2 4 4} & \mathbf{8 5 . 0 8 6 2} & \mathbf{7 9 . 4 7 5 1} & \mathbf{6 4 . 9 8 8 6} & \mathbf{7 3 . 5 3 2 1} & \mathbf{7 8 . 7 8 1 6} & \mathbf{8 2 . 5 8 7 9} & \mathbf{8 5 . 1 9 7 1} & \mathbf{8 6 . 4 4 7 1} \\
\mathbf{9 0 . 0 9 4 3} & \mathbf{9 1 . 0 1 3 0} & \mathbf{9 0 . 3 8 2 0} & \mathbf{8 7 . 7 8 0 2} & \mathbf{8 1 . 8 7 6 9} & \mathbf{6 6 . 3 2 9 1} & \mathbf{7 5 . 4 0 8 2} & \mathbf{8 0 . 9 8 7 3} & \mathbf{8 5 . 0 4 5 2} & \mathbf{8 7 . 8 4 4 3} & \mathbf{8 9 . 2 0 6 7} \\
\mathbf{9 0 . 3 9 2 0} & \mathbf{9 1 . 3 8 5 6} & \mathbf{9 0 . 7 9 4 6} & \mathbf{8 8 . 1 9 0 1} & \mathbf{8 2 . 2 0 9 0} & \mathbf{6 6 . 3 6 0 1} & \mathbf{7 5 . 5 9 8 1} & \mathbf{8 1 . 2 7 7 2} & \mathbf{8 5 . 4 1 3 4} & \mathbf{8 8 . 2 7 3 8} & \mathbf{8 9 . 6 7 4 6}
\end{array}\right)
$$

In this example, the equation $A X B=C$ is not consistent, by using Iteration method 2.1 , we can obtain the least-norm least-squares solution of the equation as

$$
X=\left(\begin{array}{ccccccccccc}
0.1013 & 0.0513 & 0.0369 & 0.0279 & 0.0234 & 0.0032 & 0.0155 & 0.0138 & 0.0135 & 0.0116 & 0.0108 \\
0.0514 & 0.0347 & 0.0293 & 0.0239 & 0.0229 & -0.0040 & 0.0138 & 0.0123 & 0.0129 & 0.0108 & 0.0102 \\
0.0353 & 0.0269 & 0.0269 & 0.0237 & 0.0264 & -0.0194 & 0.0128 & 0.0114 & 0.0135 & 0.0108 & 0.0104 \\
0.0270 & 0.0219 & 0.0241 & 0.0219 & 0.0231 & -0.0207 & 0.0116 & 0.0105 & 0.0128 & 0.0102 & 0.0099 \\
0.0227 & 0.0194 & 0.0300 & 0.0358 & 0.0839 & -0.1108 & 0.0118 & 0.0075 & 0.0197 & 0.0116 & 0.0118 \\
0.0074 & 0.0053 & -0.0262 & -0.0341 & -0.0952 & 0.2294 & 0.0003 & 0.0036 & -0.0190 & -0.0058 & -0.0079 \\
0.0152 & 0.0134 & 0.0136 & 0.0121 & 0.0123 & -0.0018 & 0.0086 & 0.0081 & 0.0085 & 0.0074 & 0.0072 \\
0.0136 & 0.0122 & 0.0130 & 0.0116 & 0.0120 & -0.0042 & 0.0082 & 0.0077 & 0.0084 & 0.0072 & 0.0070 \\
0.0122 & 0.0111 & 0.0121 & 0.0108 & 0.0113 & -0.0047 & 0.0077 & 0.0073 & 0.0080 & 0.0069 & 0.0067 \\
0.0112 & 0.0103 & 0.0119 & 0.0107 & 0.0116 & -0.0076 & 0.0074 & 0.0071 & 0.0080 & 0.0068 & 0.0067 \\
0.0103 & 0.0096 & 0.0111 & 0.0100 & 0.0108 & -0.0075 & 0.0071 & 0.0068 & 0.0077 & 0.0065 & 0.0064
\end{array}\right)
$$

where, $\sigma_{r} / \sigma_{1}=\lambda_{s} / \lambda_{1}=4.4456 / 81.1530, t=7.6710, k=7272$.

By using preconditioned method as Example 1, we can obtain the same result, but where, $\sigma_{\tau} / \sigma_{1}=\lambda_{s} / \lambda_{1}=4.1228 / 5.1832, t=0.0200, k=17$.

From the above two examples, we see that the converge rate of the iteration method is surely related to the singular value of the matrices $A, B$, and by using preconditioning methods which increase the ratio $\left(\sigma_{r} \lambda_{s}\right) /\left(\sigma_{1} \lambda_{1}\right)$, we can obtain a faster iteration rate.

\section{REFERENCES}

[1] A. Bjerhammer, 'Rectangular reciprocal matrices with special reference to geodetic calculations', Kung. Tekn. Hogsk. Handl. Stockhol 45 (1951).

[2] K-W.E. Chu, 'Symmetric solutions of linear matrix equations by matrix decompositions', Linear Algebra Appl. 119 (1989), 35-50.

[3] H. Dai, 'On the symmetric solutions of linear matrix equations', Linear Algebra Appl. 131 (1990), 1-7.

[4] F.J.H Don, 'On the symmetric solution of a linear matrix equation', Linear Algebra Appl. 93 (1987), 1-7. 
[5] G. Li, Common matrix theries and methods (Shanghai Science and Technology Press, Shanghai 1983).

[6] J.R. Magnus, 'L-structured matrices and linear matrix equation', Linear and Multilinear Algebra 14 (1983), 67-88.

[7] S.K. Mitra, 'Common solutions to a pair of linear matrix equations $A_{1} X B_{1}=C_{1}$ and $A_{2} X_{2}=C_{2}$ ', Proc.Cambridge Philos. Soc. 74 (1973), 213-216.

[8] G.R. Morris and P.L. Odell, 'Common solutions for $n$ matrix equations with applications', J. Assoc. Comput. Mach. 15 (1968), 272-274..

[9] Y.X. Peng and X.Y. Hu, 'An iteration method for the symmetric solutions and optimal approximation solution of the matrix equation $A X B=C^{\prime}$, Appl. Math. Comput. 160 (2005), 163-167.

[10] R. Penrose, 'A generalized inverse for matricies', Proc. Cambridge Philos. Soc. 5 (1955), 406-413.

[11] X.C. He and W.Y. Sun, Introduction to generalized inverse of matrices (Jiangsu Science and Technology Press, Nanjing, 1991).

College of Mathematics and Econometrics

Hunan University

Changsha 410082

Peoples Republic of China

e-mail: khguo@126.com 